\title{
La evaluación y mejora del proceso de enseñanza-aprendizaje de las matemáticas a través de la metacognición
}

\author{
Annemie Desoete \\ Departamento de Psicología Experimental Clínica y de Salud, \\ Ghent University \& Arteveldehogeschool
}

\section{Bélgica}




\section{Resumen}

Introducción. A pesar de todo el énfasis en la metacognición, los investigadores actualmente usan diferentes técnicas para evaluar la metacognición. El objetivo de esta contribución es el de ayudar a aclarar algunos de los paradigmas en la evaluación de la metacognición. El presente trabajo revisará además los estudios que intentan mejorar el proceso de aprendizaje a través de la metacognición.

Método. Se realizó un estudio longitudinal con 32 niños para investigar el aprendizaje matemático y las habilidades metacognitivas en $3^{\circ}$ y $4^{\circ}$ curso de la educación primaria. Las habilidades metacognitivas fueron evaluadas a través de las calificaciones de los profesores, protocolos de pensamiento en voz alta, calificaciones infantiles del pasado y del futuro y la EPA 2000. Describimos además algunas maneras de optimizar el aprendizaje de las matemáticas a través de la metacognición.

Resultados. A la hora de reflexionar sobre los resultados de este trabajo, existe evidencia de que la forma de evaluar determina lo que se va a conseguir. Cuestionarios infantiles no parecen reflejar sus habilidades en sí, pero son útiles para evaluar el 'conocimiento' y las 'creencias' metacognitivos de los niños. Los análisis de protocólos del pensamiento en voz alta resultaron ser técnicas precisas para evaluar las 'habilidades' metacognitivas de los niños con un nivel adecuado de fluidez verbal, pero requieren un tiempo excesivo. Los cuestionarios para los profesores muestran cierto valor añadido en la evaluación de las habilidades metacognitivas. Los datos mostraron que las habilidades metacognitivas evaluadas por calificaciones de profesores explicaron el $22,2 \%$ del rendimiento en las matemátics.

Conclusión. Sugerimos que los profesores que estén interesados en la metacognición infantil empleen diseños de métodos múltiples, incluyendo los cuestionarios a profesores para obtener una visión completa de las habilidades metacognitivas. Teniendo en cuenta la naturaleza complicada del aprendizaje matemático, puede ser útil evaluar las habilidades metacognitivas en niños para enfocarse en estos factores y su función en el aprendizaje y desarrollo matemático. Los estudios también revelan que la metacognición puede ser entrenada y tiene algo de valor añadido en la intervención con niños en la resolución de problemas matemáticos. Nuestros datos sugieren que las habilidades metacognitivas deben ser enseñadas explícitamente 
para poder conseguir una mejora y no se puede suponer que se desarrollen por una experiencia libre de las matemáticas. Puede ser posible que al dedicar más tiempo a la instrucción metacognitiva, el proceso de enseñanza-aprendizaje mejoraría.

Palabras clave: metacognición, evaluación, mejora, calificaciones del profesor, protocolo del pensamiento en voz alta, cuestionarios.

Recepción: 20-05-07 Aceptación Provisional: 08-10-07 Aceptación definitiva: 23-11-07 